\title{
Practitioner Driven Action Research... Created by a Practitioner, for Practitioners!
}

By

Michael Donahue, University of South Florida

$\mathrm{P}$ ractitioners across the United States Special Operations Command (USSOCOM) enterprise routinely engage in tackling complex organizational problems and operational deficiencies daily. Although not part of any formal creed or special operations ethos, the old saying of "if you want it done right, may as well do it yourself" resonates across the cultural mindset of the special operations enterprise. Being prepared to engage, observe, anticipate, and respond to operational challenges swiftly can put into motion successful interventions to address both shortand long-term problems. Action Research is a practitioner-driven research approach that is problem-centric and action-oriented. It simultaneously engages scientific research, problem-solving, and active learning to foster innovation and change into organizations. The task of designing and executing any research proj- ect can be quite daunting to practitioners with little or no academic research acumen. These concerns led to researching and creating a practitioner-driven framework grounded in Action Research to assist practitioners in approaching immediate problem-solving more as "practitioner-researchers." This framework is called the Practitioner Driven Action Research (PDAR) and was designed, field-tested, and then validated search (PDAR) focuses on immediate problem-solving and driving practitioners towards adopting action-driven outcomes grounded in Action Research. during a yearlong USSOCOM Action Research project to improve an operational deficiency related to military cyberspace operations. The creation of PDAR focused on addressing two questions; RQ1: What practitioner developed artifact can be constructed to help USSOCOM approach immediate problem solving more as practitioner-researchers? RQ2: How can the artifact help bridge academia and practice within USSOCOM?

Keywords: Action Research, Organizational Problem Solving, Mixed Methodology, Quantitative Research

Copyright $\odot 2020$, Michael Donahue. This article is published under a Creative Commons BY-NC license. Permission is granted to copy and distribute this article for non-commercial purposes, in both printed and electronic formats 


\section{Findings}

The research from the first data phase consisted of qualitative in vivo data coding and indicated that military practitioners are most motivated to address organizational problems collectively with peers and placed a significantly high value on opportunities for professional development in developing their research skills towards operational problem-solving. The data also indicated the most limiting factor among military practitioners in supporting Action Research initiatives within their organizations is their lack of research skills for conducting basic research. Data also showed that field practitionerswhen asked about a framework to assist them in the field-significantly favored an operationally-focused artifact using practitioner language and utilizing a step-by-step format. Interviewed practitioners also preferred an artifact with the flexibility to address a wide range of organizational problem sets. Findings from the second data collection phase-the table- top exercise-sought to validate phase one findings and measure the utility of the refined PDAR framework (see Figure 1). Practitioners applied the PDAR framework to a real-world operational problem. Direct observations indicated the six-phase framework provided practitioners much-needed structure in helping develop an initial Action Research plan. The inclusion of three Academic Advisory Group sessions during the PDAR phases offered practitioners direct access to academic expertise. The post-exercise worksheet consisted of six semi-structured questions asking participants to rank order the five most common motivational and limiting factors in supporting Action Research measured during the first phase. The elements were in random order as to not induce any bias into their ranking. The aggregated findings indicated these practitioners also ranked problem-solving collectively with peers and lack of research skills as the most motivational and limiting factors, respectively.

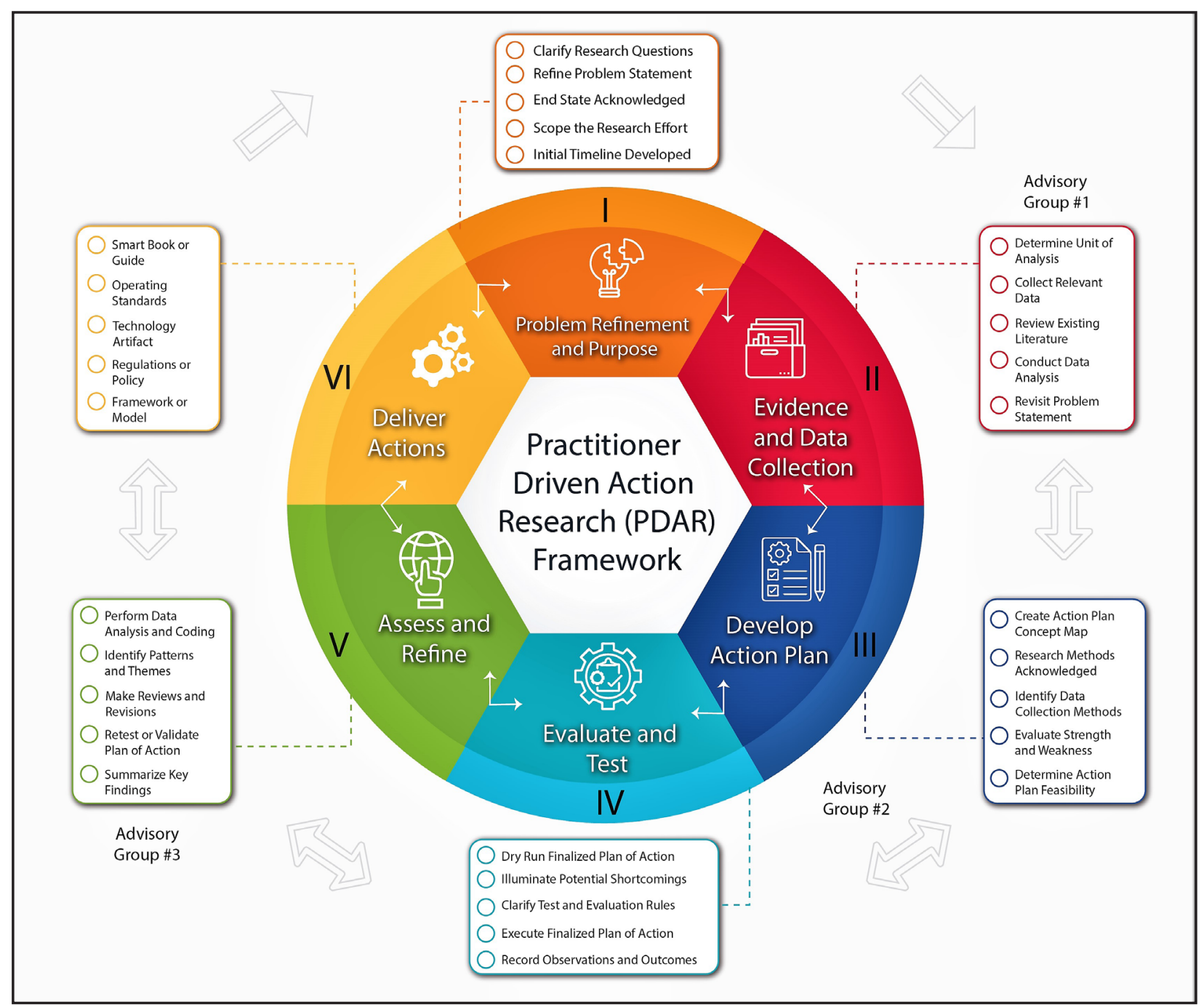

Figure 1: PDAR Framework (Version 6) 


\section{Methodology}

A mixed-method approach was used during two data collection phases. The first data phase consisted of semi-structured interviews with 32 field practitioners geographically located across six organizations that participated in the initial yearlong USSOCOM Action Research project. Twenty-five hours of interviews and 75 pages of transcript notes were collected for data analysis. The second data phase occurred six months after the conclusion of the initial Action Research project. It consisted of direct observations during a table-top exercise with 20 field practitioners applying PDAR to a real-world problem set and a semi-structured worksheet composed of six questions to help validate data from the first collection phase and assess the overall utility of the refined PDAR framework in creating an Action Research plan.

\section{Conclusions}

PDAR was initially intended to assist USSOCOM practitioners in adopting Action Research to address immediate problem-solving in their organizations. Since then, the framework endured six iterative design cycles over 18 months, culminating into a viable research framework to support organizational problem-solving across any public and private industry discipline.

Data analysis from a mixed methodology approach during two collection phases yields measurable insight into better understanding the motivational and limiting factors among field practitioners in adopting Action Research to address immediate problem-solving. Practitioners appear to have higher confidence in approaching organizational problem-solving more scientifically when given a practitioner-focused research tool to assist them. Also, built-in Academic Advisory Group sessions instill practitioner confidence in developing research skills, strengthen research rigor, and mutually beneficial to both academia and practice. Building a culture of belief among field practitioners in conducting meaningful research to address everyday problem-solving and adopting Action Research can enrich an organization's ability to tackle organizational issues and think about "how" to address them rather than merely identifying them.

\section{Where to Find Out More}

The PDAR framework presented in this research summary is part of a portfolio of work to satisfy the author's dissertation requirements for the Doctor of Business Administration, Muma College of Business, the University of South Florida. A part of the final portfolio is the publication of a practitioner smart book intended for field use in aiding practitioners in designing and implementing Action Research within their organizations. For a copy of this smart book, please contact the author at mjdonahue@outlook.com

\section{Review}

This article was accepted under the constructive peer review option. For futher details, see the descriptions at:

http://mumabusinessreview.org/peer-review-options/

\section{Author}

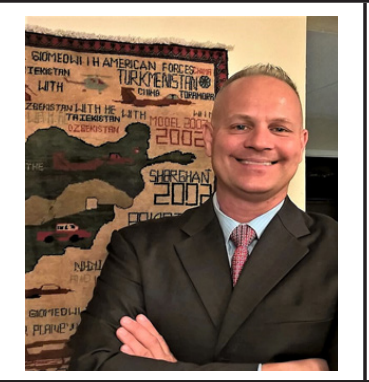

Michael Donahue is a faculty member in the Center for Advanced Studies, Joint Special Operations University, United States Special Operations Command, Tampa, FL. He served in the United States Air Force for 21 years and successfully defended his dissertation for the Doctor of Business Administration Program at the University of South Florida in 2019. He also holds master's degrees in International Relations from Troy University and Business Administration from Embry-Riddle Aeronautical University. Dr. Donahue is also an adjunct professor for the Master of Science Program in Counterterrorism at Nichols College, Dudley, MA. 